\title{
Rapid Balmer Line Variability in Ap Stars
}

\author{
H. JOHN WOOD (ESO-Chile)
}

\section{Introduction}

It is the purpose of this paper to briefly summarize the results of an eight-year obser. vational program on the Balmer-line behavior of magnetic and related stars. In addition, new data on the interesting 79-minute variable SX Phe will be presented.

\section{The Early Photometry}

The program was initiated at Kitt Peak National Observatory in 1963. It was designed to test the hypothesis that the magnetic stars show light and spectrum changes because they are pulsating. The technique employs the fact that the temperature variation of the equivalent widths of the Balmer lines reaches a rather flat maximum in the early A-type stars due to the competing effects of excitation and ionization. The Balmer lines are thus quite sensitive, via the Stark effect to pressure changes in the atmosphere. At the same time, they show little temperature sensitivity.

In the early studies, we expected to see periods of about two hours, the obtained from the period-mean density relation for main-sequence A-type stars. Indeed, KARL RAKOS found UBV periods of that duration for a number of Ap stars observed at Lowell Observatory.

Nevertheless, the results of the H-beta photometry were quite different from our expectations. The "quasi-periods" were much shorter for the hydrogen lines than for the UBV continuum.

H-beta, observed with the narrow/wide filter pairs of the CRAWFORD-STROMGREN photometry, shows a sinusoidal variation lasting for three to five cycles with periods in the range 3 to 50 minutes.

Simultaneous observations of 73 Dra in three Balmer lines showed H-beta and H-delta in phase with H-gamma opposite during part of the 20-day Eu II period. Half a period later, all three lines were seen to be in phase. See P.A.S.P., 76, 158, 1964, and A.J., 71, 378, 1966, for some results on 73 Dra.

HD 224801 (HR 9080) shows a 35-minute oscillation in H-beta on occasion. However, the period changes slightly from night to night. RAKOS had found a $2^{\text {ho }} 4^{\mathrm{m}}$ period in UBV for the star but the $35 \mathrm{~min}$. quasi-period never equaled a simple fraction of $2^{\text {ho }} 4^{\mathrm{m}}$. Much of the early narrow-band photometry line-curve work is summarized in Ap.J., 152, 117, 1968, and A.J., 72, 727, 1967.

\section{New Digital Scanner Tedniques}

In order to get information on the location, wing or line-core, of the Balmer line variations, a digital spectrum scanner was built and used at Cerro Tololo Interamerican Observatory to obtain line profiles of Beta Carinae, a bright southern A-type star.

Repeated profiles were obtained on 9 nights using the Tololo 60-inch reflector and our digital scanner. Ratios of core, wall, wing, and continuum channels were formed using some standard continuum as a reference. The individual scans had one percent fractional accuracy and the continuum/continuum ratios showed the stability of the technique. Only the extreme core channels ( $13 \AA$ band) showed the sinusoidal oscillation.

Beta Car showed quasi-periods of 33.6, 33.6, 29.4, and 36.0 minutes on four of the nine nights. The variation either lasted throughout the five-hour nightly observing run or died out with reducing amplitude. The work is described in Astronomy and Astrophysics, 12, 468, 1971. 


\section{Line Photometry of SX Phe}

Observations with wide/narrow filter pairs on $\mathrm{H}$-beta, $\mathrm{H}$-gamma, and the $\mathrm{K}$-line of ionized Calcium in SX Phe were carried out in October 1970 with the one-meter telescope and photometer of the European Southern Observatory in Chile. SX Phe has a fundamental light variation of 79 minutes, another roughly seven times that, and an extremely rapid variation of the order of a few minutes first discovered by F. B. WOOD. The observations were carried out in cooperation with a group of astronomers at Tololo headed by J. STOCK who were observing the star simultaneously.

We report here the basic observational data on hand at E.S.O. Final discussion awaits further analysis of all the data. We have $\mathrm{H}$-beta line curves on three nights covering seven cycles, a H-gamma line curve covering 1.5 cycles, and a $\mathrm{K}$ line-curve covering three cycles. The time resolution of all these observations is excellent: of the order of one observation every 15 seconds.

$\mathrm{H}$-beta and $\mathrm{H}$-gamma vary strongly in equivalent width (max. range 2.70 to 2.88 mag in $\beta$ ) and are in phase with each other. The $\mathrm{K}$-line is opposite in phase to the Balmer lines and seems to mimic the color/temperature behaviour of the star. The Balmer line-curves are remarkably similar in shape to the light-curves. Thus the light of the star seems to vary with the pressure changes in the stellar atmosphere.

The very rapid oscillations seen in the UBV light-curves seem also to exist in the Balmer line-curves.

Further analysis of these accurate observations should provide a wealth of new information about this remarkable star. Papers on SX Phe by STOCK and TAPIA have recently appeared in: Astronomy and Astrophysics, 10, 134, and 10, 147, 1971, and in A. and A. Supplement Series, 3, 253, 1971 .

\section{Conclusion}

The rapid Balmer-line variations seen in the several stars discussed above cannot be described as a simple Eddington-type pulsation of the star. A more attractive interpretation of the phenomenon comes from the hydrodynamics of gravo-acoustic waves. Calculations based on the acoustic-wave theory by MOORE and SPIEGEL give periods on the order of 30 minutes for A-star atmospheres. This theory satisfactorily explains the 5 -minute oscillations of the solar atmosphere driven by the mechanical energy of convection. It is our contention that these same frequencies are excited in the Ap stars by explosive flare-like activity in the stellar atmosphere.

The acoustic frequencies depend on the local value of the effective gravity. Since the gravity varies with latitude in a rotating star, quasi-periods will be different depending on the latitude of the exciting flare. In the case of Beta Carinae, the value of $v \sin i$ is $210 \mathrm{~km} / \mathrm{sec}$. Thus one can understand how the periods can range between 29 and 36 minutes.

The above hypothesis needs checking with detailed hydro-dynamic calculations and with observations in Lyman $\alpha$ or $\mathrm{H} \alpha$. The exciting flare may be visible in emission in these lines if sufficient time and spectral resolution is available.

The author wishes to thank the National Science Foundation (U.S.A.) for support of this work while he was at McCormick Observatory. 